\title{
Wind-Turbine Wakes in a Convective Boundary Layer: A Wind-Tunnel Study
}

\author{
Wei Zhang · Corey D. Markfort • Fernando Porté-Agel
}

Received: 30 September 2011 / Accepted: 18 June 2012 / Published online: 15 July 2012

(C) Springer Science+Business Media B.V. 2012

\begin{abstract}
Thermal stability changes the properties of the turbulent atmospheric boundary layer, and in turn affects the behaviour of wind-turbine wakes. To better understand the effects of thermal stability on the wind-turbine wake structure, wind-tunnel experiments were carried out with a simulated convective boundary layer (CBL) and a neutral boundary layer. The CBL was generated by cooling the airflow to $12-15^{\circ} \mathrm{C}$ and heating up the test section floor to $73-$ $75^{\circ} \mathrm{C}$. The freestream wind speed was set at about $2.5 \mathrm{~m} \mathrm{~s}^{-1}$, resulting in a bulk Richardson number of -0.13 . The wake of a horizontal-axis 3-blade wind-turbine model, whose height was within the lowest one third of the boundary layer, was studied using stereoscopic particle image velocimetry (S-PIV) and triple-wire (x-wire/cold-wire) anemometry. Data acquired with the S-PIV were analyzed to characterize the highly three-dimensional turbulent flow in the near wake (0.2-3.2 rotor diameters) as well as to visualize the shedding of tip vortices. Profiles of the mean flow, turbulence intensity, and turbulent momentum and heat fluxes were measured with the triple-wire anemometer at downwind locations from 2-20 rotor diameters in the centre plane of the wake. In comparison with the wake of the same wind turbine in a neutral boundary layer, a smaller velocity deficit (about $15 \%$ at the wake centre) is observed in the CBL, where an enhanced radial momentum transport leads to a more rapid momentum recovery, particularly in the lower part of the wake. The velocity deficit at the wake centre decays following a power law regardless of the thermal stability. While the peak turbulence intensity (and the maximum added turbulence) occurs at the top-tip height at a downwind distance of about three rotor diameters in both cases, the magnitude is about $20 \%$ higher
\end{abstract}

W. Zhang · C. D. Markfort

Saint Anthony Falls Laboratory, Department of Civil Engineering, University of Minnesota, Minneapolis, MN 55414, USA

e-mail: wzhang@umn.edu

C. D. Markfort

e-mail: mark0340@umn.edu

F. Porté-Agel ( $\varangle)$

Wind Engineering and Renewable Energy Laboratory (WIRE), École Polytechnique Fédérale de Lausanne (EPFL), EPFL-ENAC-IIE-WIRE, 1015 Lausanne, Switzerland

e-mail: fernando.porte-agel@epfl.ch 
in the CBL than in the neutral boundary layer. Correspondingly, the turbulent heat flux is also enhanced by approximately $25 \%$ in the lower part of the wake, compared to that in the undisturbed CBL inflow. This study represents the first controlled wind-tunnel experiment to study the effects of the CBL on wind-turbine wakes. The results on decreased velocity deficit and increased turbulence in wind-turbine wakes associated with atmospheric thermal stability are important to be taken into account in the design of wind farms, in order to reduce the impact of wakes on power output and fatigue loads on downwind wind turbines.

Keywords Atmospheric boundary layer - Convective boundary layer · Thermal stability · Wind-tunnel experiment · Wind-turbine wakes

\section{Introduction}

In spite of the important advances made in the aerodynamic design of wind turbines, the complex interaction of turbines with the highly turbulent flow in the atmospheric boundary layer (ABL) is not fully understood. Detailed information about wind-turbine wake properties, including mean flow characteristics and turbulence structures, is of great importance for optimizing wind-turbine siting, maximizing the energy production in wind farms and ensuring structural integrity of wind turbines in the planning of wind-farm projects. Turbulent wakes downwind of horizontal-axis wind turbines have been studied extensively, and comprehensive reviews of the aerodynamics of wind-turbine wakes can be found in Vermeer et al. (2003) and Sørensen (2011). Wind-turbine wakes are characterized by reduced mean wind speeds (velocity deficit), higher wind shear and increased turbulence levels compared to the undisturbed incoming flow, either in a uniform freestream or boundary-layer type flows.

Wind turbines operate in the lowest region of the atmospheric boundary layer. As a result, wind-turbine wakes are affected by the same factors that influence the turbulent boundary layer, including surface inhomogeneities (roughness and topography) and thermal stratification. The effects of the incoming flow on the wind-turbine wake structure have been studied through wind-tunnel experiments and numerical simulations (e.g., Whale et al. 2000; Vermeer 2001; Medici and Alfredsson 2006; Chamorro and Porté-Agel 2009, 2010; Hancock and Pascheke 2010; Porté-Agel et al. 2011; Wu and Porté-Agel 2011). It should be noted that incoming flow conditions become particularly important for the modern tall wind turbines, which usually have rotor diameters of more than $100 \mathrm{~m}$. In those cases, the flow cannot be considered uniform over the rotor-swept area and, consequently, the variation of wind speed and turbulence across the rotor must be taken into account.

The atmospheric boundary layer is usually classified into three types: neutral, convective and stable, according to the thermal stratification and the dominant mechanisms of turbulence generation. In a neutral boundary layer, the mean potential temperature gradient is negligible and the production of turbulence is dominated by the strong shear near the surface. The atmosphere is neutral for only a limited amount of the time, occurring either in the morning or evening, during the transition between stable and unstable conditions, or when skies are overcast with moderate to high wind speeds (under which a small temperature gradient exists between the ground and the air above). The unstable or convective boundary layer (CBL) is common in fair, sunny weather, and anytime when the ground is warmer than the air flow, and also when upside-down convection is generated by cloud-top radiative cooling (Garratt 1994). In the CBL, the turbulent kinetic energy (TKE) and the vertical transport of momentum, heat and moisture are enhanced by positive buoyancy (Stull 1988). Thermal stratification modifies the wind profiles of boundary-layer flows. Over a flat surface, the mean 
wind profile of the surface layer (the lowest 10-15\% of a fully developed turbulent boundary layer) follows the logarithmic law modified by a stability correction. It can be written as

$$
U(z)=\frac{u_{*}}{\kappa}\left[\ln \left(\frac{z}{z_{0}}\right)-\psi_{m}\left(\frac{z}{L}\right)\right]
$$

where $u_{*}$ is the friction velocity, $\kappa$ is the von Karman constant $(\approx 0.4), z_{0}$ is the aerodynamic roughness length and $\psi_{m}$ is a function of the dimensionless height $z / L$. The Obukhov length $L$ is defined as $L=\frac{-\bar{\theta} u_{*}^{3}}{\kappa g\left(\overline{w^{\prime} \theta^{\prime}}\right)_{s}}$, where $\bar{\theta}$ is the reference temperature, $g$ is gravitational acceleration, and $\overline{\left(w^{\prime} \theta^{\prime}\right)_{s}}$ is the surface kinematic heat flux. Due to the positive or negative buoyancy contributing to the production or destruction of turbulence, turbulence intensity is a function of atmospheric thermal stratification as well as surface roughness. The thermal stability can be quantified by the gradient Richardson number $R i\left(=\frac{g}{\bar{\theta}} \frac{\partial \theta / \partial z}{(\partial U / \partial z)^{2}}\right)$, which is negative for the unstable or convective boundary layer, zero for the neutral case and positive for the stable case.

Field measurements of wind-turbine wakes at wind farms are affected by seasonal and diurnal variations, which reflect the intrinsic non-stationarity of the atmosphere. Evidence of significant thermal stability effects on the wind-turbine power output has been reported in full-scale field measurements. van den Berg (2008) argued that the thermal stability in a stable boundary layer increases the wind shear and hence substantially increases the power production compared to a neutral boundary layer. Recently, Wharton and Lundquist (2010) found that, for a given wind speed, less power is produced by turbines during convective than stable conditions. The power output difference was found to be as large as $20 \%$ between the stable and convective regimes. In addition, wind-turbine wake structures are affected by the thermal stratification of the boundary layer. Baker and Walker (1984) carried out measurements behind a $2.5-\mathrm{MW}$ wind turbine $(d=91 \mathrm{~m})$ at Goodnoe Hills, Washington mainly in stable nighttime conditions. Their results indicated that the wake velocity deficits were on the order of $15-18 \%$ at nine rotor diameters downwind and as high as $30-40 \%$ at three to five rotor diameters. Under higher-level turbulent flow conditions, velocity deficits were decreased to less than $10 \%$. Magnusson and Smedman (1994) reported wind-turbine wakes measured at 4.2, 6.1 and 9.6 rotor diameters downwind of a wind turbine in the Alsvik wind farm. For unstable stratification, with $R i<-0.05$ estimated with the mean temperature gradient over the rotor-swept plane, the velocity deficit was shown to be independent of stability. However, the velocity deficit increased linearly in the weakly unstable and weakly stable conditions $(-0.05<R i<0.05)$. These authors reported later that the velocity deficit tended to decrease under stable conditions with $R i>0.25$, due to more effective turbulent mixing caused by breaking waves and turbulent bursts (Magnusson and Smedman 1999). As illustrated above, atmospheric thermal stability cannot be viewed as a small perturbation to a basic neutral state when studying tall wind turbines (van den Berg 2008); rather, thermal stability and its induced flow variation should be accounted for in understanding turbulent wind-turbine wakes.

Wind-tunnel experiments have been widely used to study the atmospheric boundary layer. Specifically, physical simulations of thermally stratified boundary layers have been carried out by e.g., Meroney and Melbourne (1992), Fedorovich et al. (1996), Ohya (2001), Ohya and Uchida (2004), Chamorro and Porté-Agel (2010), and Hancock and Pascheke (2010). Recently, Chamorro and Porté-Agel (2010) studied the effect of a stable boundary layer (SBL) on the wake structure of a stand-alone wind turbine. They found that, in a relatively weak stable case (the bulk Richardson number $R i_{b}=\frac{g}{\theta} \frac{\delta \Delta \theta}{U_{0}^{2}}=0.15$, where $\Delta \theta$ is the temperature difference between the freestream flow and the surface), the maximum velocity deficit 
is located at the hub height and follows a power-law decay with distance from the turbine. The stronger shear of the incoming SBL flow leads to a slightly stronger and larger region of enhanced turbulence intensity, extending between three and six rotor diameters compared with four to five rotor diameters in the neutral boundary layer. Additionally, Hancock and Pascheke (2010) investigated the effect of a moderately stable ABL on wind-turbine wakes, and found a larger maximum velocity deficit in the near wake region, a reduced wake height and increased width in the far wake in the stable case compared to the neutral case. To our best knowledge, there is no report of wind-tunnel tests of wind-turbine wakes in a CBL, which often occurs overland during the day.

Our study aims to quantify the effects of the CBL on the wind-turbine wake, including tip vortices, velocity deficit and turbulence characteristics, compared to that in a neutral boundary layer. We employed two measurement techniques: stereoscopic particle image velocimetry (S-PIV) to quantify the three-dimensional (3-D) turbulent near wake and visualize the coherent vortex structure, and a high temporal-resolution customized triple-wire ( $\mathrm{x}$-wires combined with a cold-wire) to conduct time series measurements of the streamwise and vertical velocities as well as temperature in the wake $(x / d=2-20)$. This study provides an opportunity to understand the effects of atmospheric stability on wind-turbine wakes using well-controlled wind-tunnel experiments. Also, the results presented here are ideal for validating and guiding the development of improved models for the turbulent fluxes of momentum and heat as well as turbine-induced forces in numerical models such as large-eddy simulation (LES).

\section{Experimental Set-Up}

\subsection{Thermally Controlled Boundary-Layer Wind Tunnel}

Experiments were carried out in the recirculating boundary-layer wind tunnel at the Saint Anthony Falls Laboratory, University of Minnesota, U.S.A. The main test section for boundary-layer flow studies has a length of $16 \mathrm{~m}$ and a cross-section of $1.7 \times 1.7 \mathrm{~m}^{2}$, with a turbulent boundary layer developed using a tripping mechanism (40-mm picket fence) at the entrance of the wind-tunnel test section. The test-section floor is made up of a series of smooth aluminum plate heat exchangers. To achieve the desired thermal stratification conditions (neutral, stable or unstable), the temperature of the test section floor and airflow can be independently controlled between $5{ }^{\circ} \mathrm{C}$ to $80{ }^{\circ} \mathrm{C} \pm 0.25^{\circ} \mathrm{C}$. The convective boundary layer was simulated with the air cooled to $\theta_{0}=10-12^{\circ} \mathrm{C}$ and the floor heated up to $\theta_{s}=73-75^{\circ} \mathrm{C}$. For the neutral boundary layer, both the air and the floor temperature were maintained at $30^{\circ} \mathrm{C}$. More details on this wind-tunnel configuration and operations can be found in Carper and Porté-Agel (2008) and Chamorro and Porté-Agel (2010).

\subsection{Measurement Methodology}

\subsubsection{Triple-Wire Anemometry}

A customized high-frequency triple-wire (combination of a x-wire and a cold-wire) was used to measure the instantaneous velocity and temperature profiles at selected streamwise locations. The probe is made of $5.0 \mu \mathrm{m}$ platinum-coated tungsten wires (x-type hot wires) and a $2.5 \mu \mathrm{m}$ wire (cold wire), which are connected to an A. A. Lab Systems AN-1003 10-channel CTA system. The sensor was mounted on a traversing system (Velmex, Inc) so that multiple positions can be accurately measured using computer control. 
The anemometer was calibrated in a well-controlled small calibration unit against a Pitotstatic probe and a thermocouple at seven inclination angles and multiple flow speeds with several temperatures. A cubic-spline table calibration method was used to determine the two instantaneous velocity components from the two instantaneous voltage signatures, and then instantaneous velocities were corrected for temperature from the cold-wire measurements. Pre- and post-experiment calibrations were carried out to check the validity of the calibration throughout the experiments. During calibration, the mean temperature variation of the airflow was kept within a range of $\pm 0.25^{\circ} \mathrm{C}$ to avoid bias errors caused by the thermal drift of the voltage signal. Velocities measured with the $\mathrm{x}$-wire were considered accurate to, at best, $1 \%$. Turbulence intensity accuracy depends on a complex combination of probe length, wallnormal positioning and Reynolds number as discussed by Jorgensen (1996) and Hutchins et al. (2009).

\subsubsection{Stereo-PIV}

A high-resolution S-PIV (TSI, Inc.) system was employed to measure the three velocity components $(u, v, w)$ of the near wake $(x / d=0.2-3.2)$ in the vertical central plane $(y=0)$. A dual-head Nd:Yag laser (Quantel, USA) was used to produce the maximum laser pulse of $190 \mathrm{~mJ}$. The laser beam was transferred by an optical arm to the wind-tunnel test section and transformed into a lightsheet using cylindrical and spherical lenses. Two PowerView Plus 4MP 12-bit CCD cameras $(2048 \times 2048$ pixels) fitted with $105 \mathrm{~mm}$ lenses were set up with Scheimpflug mountings. A home-made atomizer was used with olive oil to generate seeding particles for PIV measurements. Multiple cylindrical reservoirs were filled half-full with olive oil, and compressed air was forced through Laskin nozzles to atomize the oil. $1-\mu \mathrm{m}$ diameter oil droplets were produced and released into the entrance of the test section, where they were allowed to fill the test section and reach a uniform distribution prior to taking measurements.

Particle images were evaluated to determine instantaneous velocity fields using a multigrid method with second-order accuracy (Insight 3G, TSI). The final interrogation window is $32 \times 32$ pixels with $50 \%$ overlapping, while the corresponding measurement resolution is approximately $2.3 \mathrm{~mm} \times 2.3 \mathrm{~mm}$. The maximum residual error is 1.5 pixels. Erroneous vectors, less than $1 \%$ of the total extracted vectors, were replaced by vectors interpolated through the Gaussian scheme using good neighbouring vectors. The velocity field was obtained by ensemble-averaging 1500 instantaneous velocity fields, which ensures converged estimates of turbulence statistics. All fluctuating velocity fields were employed to calculate the spatial distributions of turbulence intensities and shear stresses. The measurement uncertainty level of the velocity vectors is estimated to be within $2 \%$.

\subsection{Experimental Details}

The miniature model wind turbine employed in this study is a functional turbine that consisted of a three-blade GWS/EP-5030 3 rotor driving a small DC generator to extract energy from the incoming boundary-layer flow. The rotor blades are nearly flat plates, similar to those of the small homemade wind-turbine models used in Lebrón J et al. (2010) and Cal et al. (2010). As shown in Fig. 1, the rotor diameter is $0.13 \mathrm{~m}$ and the tower is $0.105 \mathrm{~m}$. The bottom tip of the turbine is $0.04 \mathrm{~m}$ above the surface and the top tip is at a height of $0.17 \mathrm{~m}$, ensuring that the turbine rotor-swept area is within the lowest one third of the turbulent boundary layer. With the freestream wind speed $\left(U_{0}\right)$ of approximately $2.5 \mathrm{~m} \mathrm{~s}^{-1}$, the rotor operates at $1140-1160$ r.p.m in the neutral boundary layer and 1280-1320 r.p.m in the CBL. This gives a tip-speed 


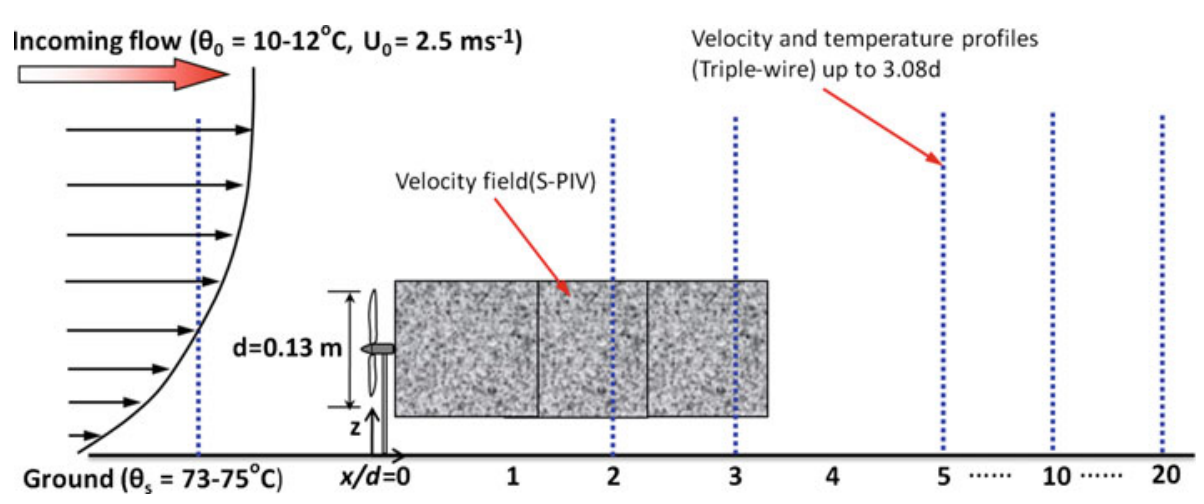

Fig. 1 Schematic diagram of the experimental set-up and the coordinate system definition downwind of a model wind turbine

Table 1 Characteristics of the neutral boundary layer and CBL

\begin{tabular}{llllllll}
\hline BL & Floor temp. $\theta_{s}\left({ }^{\circ} \mathrm{C}\right)$ & Air temp. $\theta_{0}\left({ }^{\circ} \mathrm{C}\right)$ & $z_{0}(\mathrm{~mm})$ & $u_{*}\left(\mathrm{~m} \mathrm{~s}^{-1}\right)$ & $R e$ & $R i_{b}$ & $\delta / L$ \\
\hline Neutral & 30 & 30 & 0.030 & 0.12 & 4430 & - & - \\
Convective & $73-75$ & $10-12$ & 0.026 & 0.14 & 5350 & -0.13 & -1.36 \\
\hline
\end{tabular}

ratio $\left(\lambda=\Omega(2 \pi / 60)(d / 2) / U_{\text {hub }}\right.$, where $U_{\text {hub }}$ is the incoming wind speed at the hub height $)$ of approximately 3.7 and 3.8 for the neutral boundary layer and CBL cases, respectively.

The turbulent boundary-layer depth $\delta$ is approximately $0.6 \mathrm{~m}$, determined to be the elevation where the streamwise velocity magnitude reaches $99 \%$ of the freestream speed $U_{0}$, at the measurement location for both neutral boundary layer and CBL cases. The main parameters of the simulated neutral boundary layer and CBL are summarized in Table 1. The heating and cooling scheme yields an average thermal stratification of $98.4 \mathrm{~K} \mathrm{~m}^{-1}$ within the CBL; the friction velocity $u_{*}$ is $0.14 \mathrm{~m} \mathrm{~s}^{-1}$ and the roughness length $z_{0}$ is $0.026 \mathrm{~mm}$, estimated by fitting the mean velocity profiles in the surface layer using the logarithmic law with the stability correction. Accordingly, the Reynolds number based on the friction velocity $u_{*}$ and the boundary-layer depth $\delta$ is $R e=u_{*} \delta / v=5350$; the Obukhov length $L$ is estimated to be $-0.44 \mathrm{~m}$, thus the ratio of the CBL depth to $L$ is $\delta / L=-1.36$. Also, the bulk Richardson number $R i_{b}$ is about -0.13 . In order to achieve reasonably large $R i_{b}$ within the capability of the facility, we had to perform experiments at a relatively low freestream speed while still ensuring the wind-turbine model remained operational. This leads to Re lower than that of a full-scale flow by two orders of magnitude. The influence of $R e$ on the near wake is not well understood, however, the bulk properties of the fully turbulent wake downwind of the turbine model are less sensitive to $R e$ (Whale et al. 1997). In addition, primary wake characteristics, such as wake rotation and tip vortices, can be reproduced even at relatively low $R e$ (Medici and Alfredsson 2006). This highlights the value of wind-turbine wake data obtained in wind tunnels for the purpose of understanding the fluid dynamics as well as validating and developing numerical models such as LES, Reynolds-averaged Navier-Stokes (RANS) and analytical wake models.

The vertical profiles of the inflow for both the neutral boundary layer and CBL cases are shown in Fig. 2. The normalized streamwise velocity is slightly higher in the CBL than that in the neutral boundary layer, and the maximum turbulence intensity, just above the surface, 

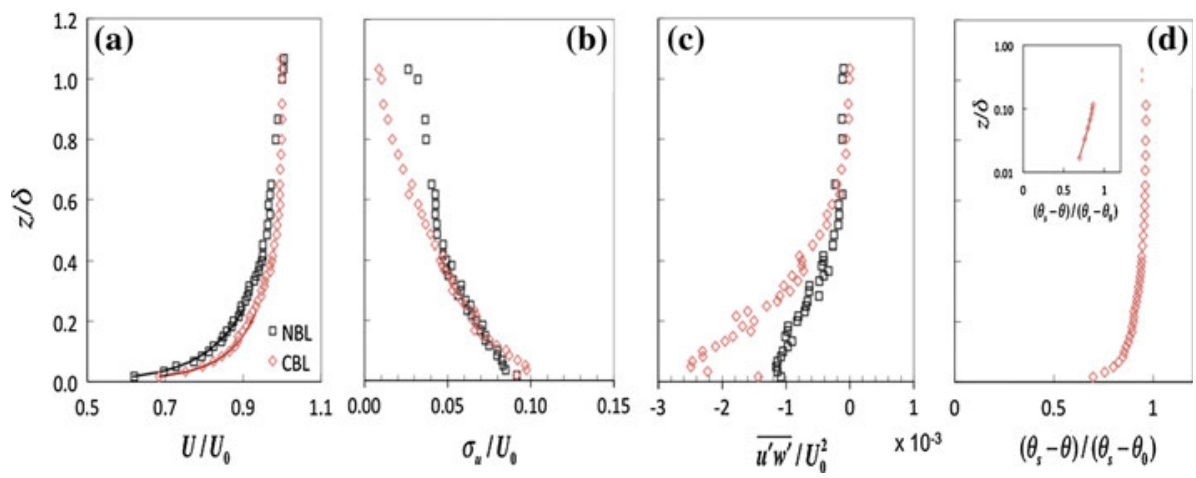

Fig. 2 Inflow profiles for the CBL and the neutral boundary layer. a Mean streamwise velocity fitted with M-O similarity theory in the surface layer; $\mathbf{b}$ streamwise turbulence intensity; $\mathbf{c}$ kinematic shear stress; $\mathbf{d}$ mean temperature with inset showing fit with M-O similarity theory in the surface layer

was $9 \%$ in the neutral boundary layer versus $11 \%$ in the CBL. As expected, stronger vertical mixing in the CBL results in a higher turbulence intensity. Similarly, the kinematic shear stress near the surface in the CBL is higher than that in the neutral boundary layer (Fig. 2c). Fitting the logarithmic law with the stability correction to the mean velocity and temperature profiles in the surface layer $(z<|L|$, in Fig. 2a, d), one can see that the surface-layer flow follows Monin-Obukhov similarity theory.

Previous wind-tunnel studies of the inversion-capped CBL (Fedorovich et al. 1996, 2001a,b and Ohya and Uchida 2004) presented the turbulence statistics normalized with convective mixed-layer scaling parameters (Deardorff 1970), including $z_{i}, w_{*}$ and $\theta_{*}$ for the length, velocity and temperature, respectively. The mixed-layer depth $z_{i}$ is defined as the height at where the heat flux reaches the minimum in the entrainment zone. Because of the lack of a capping inversion layer in the present simulated CBL, we used the boundary-layer depth $\delta$ to approximate $z_{i}$. Accordingly, $w_{*}$ and $\theta_{*}$ are calculated by $w_{*}=\left(\frac{g}{\bar{\theta}}\left(\overline{w^{\prime} \theta^{\prime}}\right)_{s} z_{i}\right)^{1 / 3}$ and $\theta_{*}=\frac{\left(\overline{w^{\prime} \theta^{\prime}}\right)_{s}}{w_{*}}$. Compared with previous wind-tunnel data in Fedorovich et al. (1996), the current flow statistics shown in Fig. 3 display an overall similar trend up to $z / \delta=0.4$, indicating that the generated boundary layer reasonably represents the lower portion of a classic CBL. Specifically, the temperature variance and the turbulent kinematic heat-flux profiles follow similar trends with comparable magnitude to those in Fedorovich et al. (1996). The departure observed in the velocity variance profiles for $z / \delta<0.4$ is mainly attributed to the difference in the thermal stability strength of the two studies. It should be noted that, however, the flow statistics beyond $z / \delta=0.4$ deviate from the previous wind-tunnel datasets due to the absence of the capping thermal inversion layer above. Considering that the wind-turbine model occupies the lowest one third of the boundary layer, the simulated CBL inflow is deemed adequate to study the effects of surface-layer thermal stability on the structure of wind-turbine wakes.

The turbulent wake downwind of the model wind turbine was measured with a combination of two techniques: S-PIV and a customized triple-wire; S-PIV provides detailed vortical structure in the near wake $(x / d=0.2-3.2)$, including helicoidal tip vortices. The triple-wire anemometer was positioned in the central plane $(y=0)$ in the wake at selected downwind streamwise locations $(x / d=2,3,5,10$ and 20). Vertical profiles were collected starting at $z=0.01 \mathrm{~m}$ up to $z=0.4 \mathrm{~m}$ with intervals of $\Delta z=0.01 \mathrm{~m}(\Delta z / d=0.08)$ between 

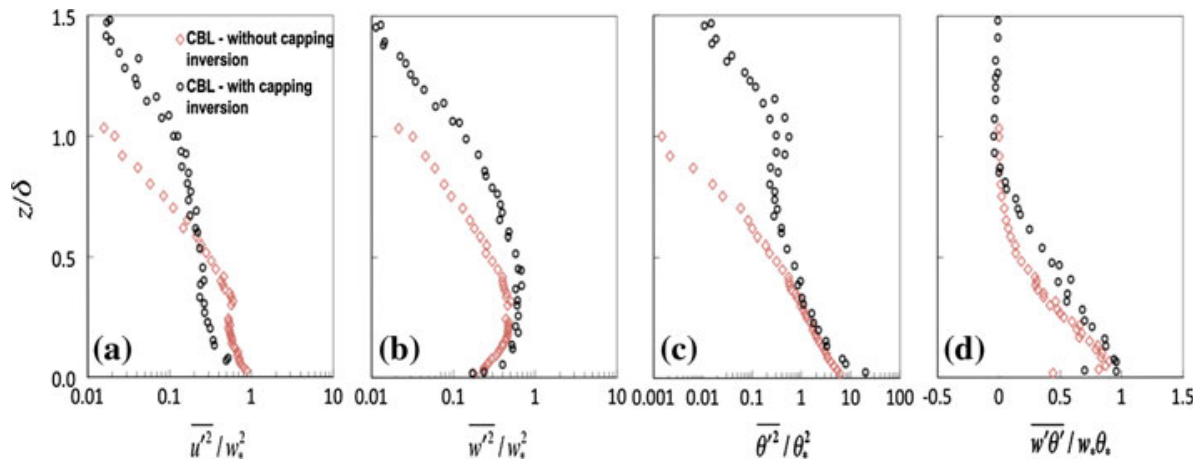

Fig. 3 Comparison of the simulated CBL without the capping inversion layer with the inversion-capped CBL in Fedorovich et al. (1996). a Streamwise velocity variance; $\mathbf{b}$ vertical velocity variance; $\mathbf{c}$ temperature variance; and $\mathbf{d}$ turbulent kinematic heat flux

measurement points. Time series of instantaneous velocities and temperature were obtained for $60-120 \mathrm{~s}$ at a frequency of $1000-2000 \mathrm{~Hz}$.

\section{Results and Discussion}

\subsection{Velocity Deficit}

Wind turbines extract kinetic energy from the incoming flow, which results in reduced velocity in the wake. The mean streamwise velocity contours at $x / d=0.2-3.2$ for the neutral boundary layer and the CBL cases are shown in Fig. 4. The near-wake is characterized by low-momentum flow, in particular, a "dead zone" with nearly zero velocity, immediately behind the nacelle (until $x / d \approx 0.6$ ). Also, Fig. 4 illustrates the highly non-symmetric velocity distribution in the vertical direction, primarily due to the boundary-layer type incoming flow and the presence of the ground surface. Though the velocity fields look very similar in both stability cases, the low-momentum flow region is noticeably smaller in the CBL case. Specifically, the velocity has a relatively higher magnitude in the lower part of the wake in the CBL compared with that in the neutral case.

Additional quantitative comparison of the normalized streamwise velocity ( $U / U_{\text {hub }}$ ) profiles with the undisturbed incoming flow for the neutral boundary layer and CBL cases is shown in Fig. 5. In general, the $U$ profiles follow a similar trend except that the magnitude is less in the neutral boundary layer than that in the CBL case. Large departures of the streamwise velocity from the inflow in the two cases are found between the bottom-tip and top-tip heights. The streamwise velocity deficit, an indicator of how rapidly the wind-turbine wake recovers to the undisturbed incoming flow, is calculated by

$$
\frac{\Delta U_{x}(z)}{U_{\text {hub }}}=\frac{U_{x}(z)-U_{0}(z)}{U_{\text {hub }}},
$$

where $U_{0}(z)$ is the streamwise velocity of the incoming flow at the vertical position $z$. Figure 5 demonstrates that the velocity deficit in the turbine wake is generally smaller in the CBL than that in the neutral case. Moreover, the departure of the velocity deficit in the two cases is primarily seen below the hub height, i.e., in the lower part of the wake. This result clearly shows the faster recovery of the wind-turbine wake in the CBL compared to the neutral case, 

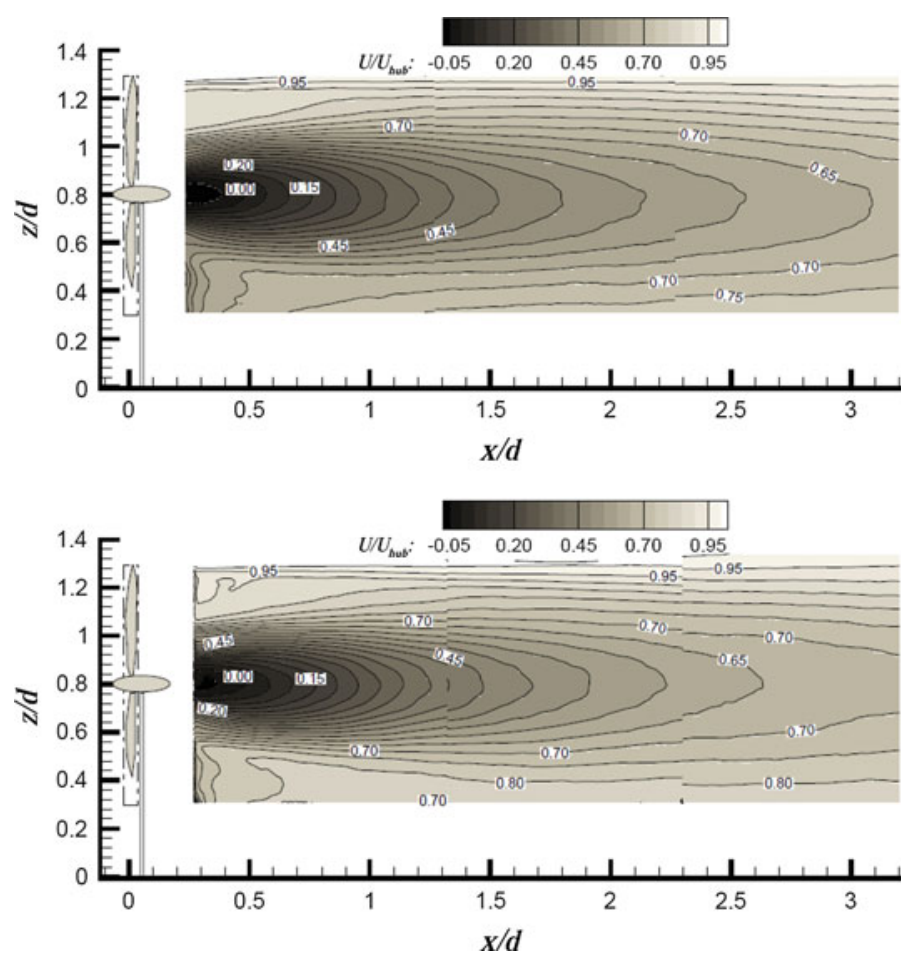

Fig. 4 The streamwise velocity at $x / d=0.2-3.2$ behind the wind-turbine model in the neutral boundary layer (upper) and in the CBL (lower)

which is attributed to the increased momentum transport and turbulent mixing induced by convection.

The streamwise velocity and/or velocity deficit at the centre of the wake (around the hub height) is an important parameter with which to estimate power production and predict overall wake-profile characteristics. With the assumption of self-similar behaviour of the wake, the wake profile can be expressed as a function of the velocity deficit at the hub height and the radial distance away from the wake centre (Frandsen et al. 2006). In this regard, it is critical to understand how the velocity deficit at the hub height varies with downwind distance. Simple empirical models have been proposed to describe the velocity deficit in the wake, following a power-law decay of the form (Vermeer et al. 2003):

$$
\frac{\Delta U_{x}}{U_{\text {ref }}}=A\left(\frac{x}{d}\right)^{n},
$$

where $U_{\text {ref }}$ is the reference velocity, either the freestream wind speed or the incident wind speed at the hub height. The range of two constants regressed from full-scale field data and wind-tunnel data is quite large; with $A \in[1,3]$ and $n \in[-1.25,-0.75]$. The variation is attributed to various sources of data collected in the field or in wind tunnels under a variety of conditions. In particular, field data must be interpreted and compared with caution, because they may be affected by heterogeneous upwind conditions and non-stationarities, including complicated topography, tall canopies, surface roughness change, transition of thermal stability, and wind-direction variability. 

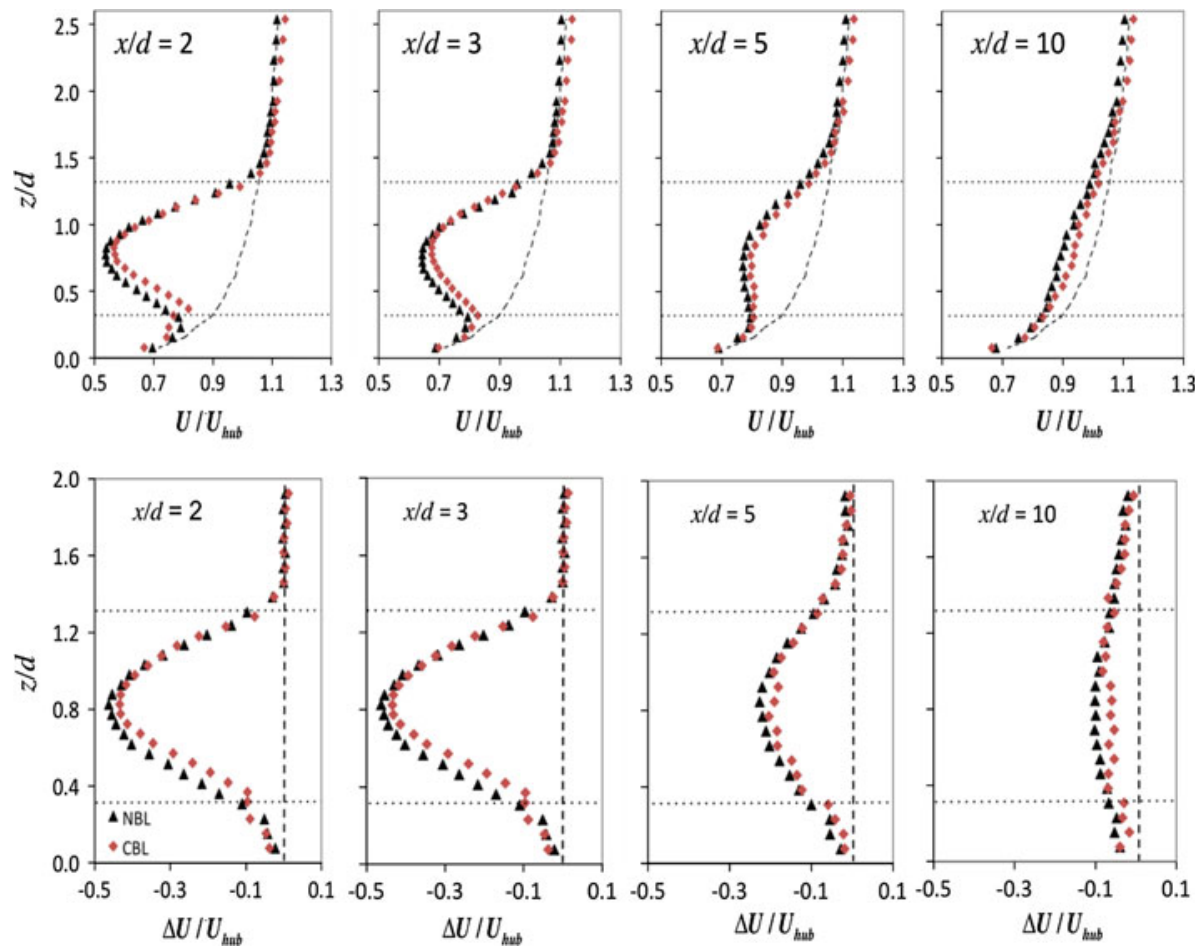

Fig. 5 Comparison of vertical profiles of the streamwise velocity (upper) and the streamwise velocity deficit (lower) downwind of the turbine to the incoming flow in the neutral boundary layer and in the CBL. The horizontal upper and lower dashed lines indicate the top-tip and the bottom-tip heights, respectively. This also applies to other similar plots

The velocity deficit at the hub height as a function of the distance from the rotor, plotted on a log-log scale (Fig. 6), shows that the velocity deficit decays roughly following a power law. The neutral boundary layer and CBL plots seem to be parallel with each other (a slightly larger slope in the CBL case), and this trend remains until $x / d=20$. This again indicates that stronger turbulent mixing causes a faster recovery of the wake in the CBL case.

The decay rate of the velocity deficit presented in Fig. 6 displays a similar behaviour to the field data of Magnusson and Smedman (1994). These curves have a slope of -0.81 (neutral boundary layer) and $-0.82(\mathrm{CBL})$ compared to -0.8 for theirs, but the magnitude of the velocity deficit is about $20-30 \%$ lower in our cases. This is likely related to the efficiency and loading conditions of the wind-turbine model employed. Wind-tunnel data reported by Hancock and Pascheke (2010) showed that the velocity deficit in the SBL was larger than that in the neutral boundary layer, which qualitatively agrees with the trend in the velocity deficit under unstable stratification effects in Magnusson and Smedman (1994). It should be remarked that coefficients $A \approx 1$ and $n \approx-1$ were used by Högström et al. (1988) and Barthelmie et al. (2004) to fit field data, implying a faster decay rate of the velocity deficit. 


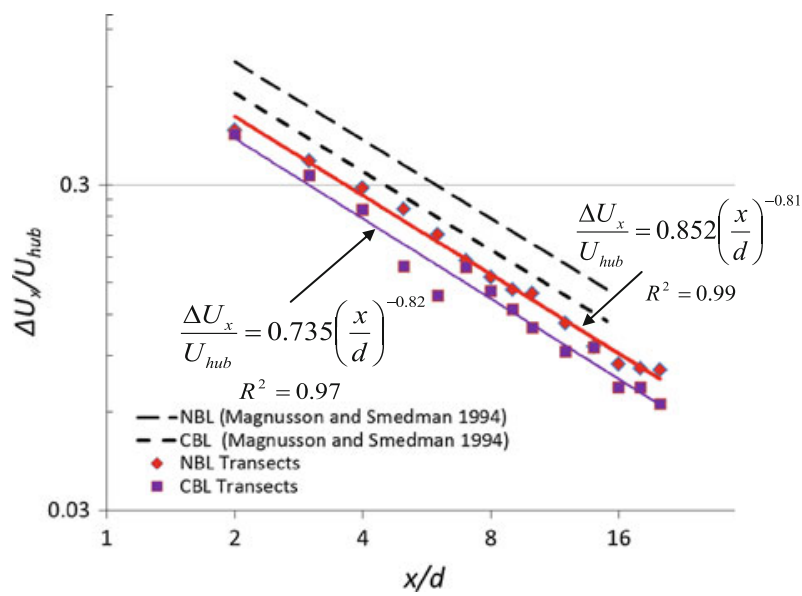

Fig. 6 The streamwise velocity deficit $\left(\Delta U_{x} / U_{\text {hub }}\right)$ at the hub height as a function of the distance from the wind turbine in the neutral boundary layer and CBL cases
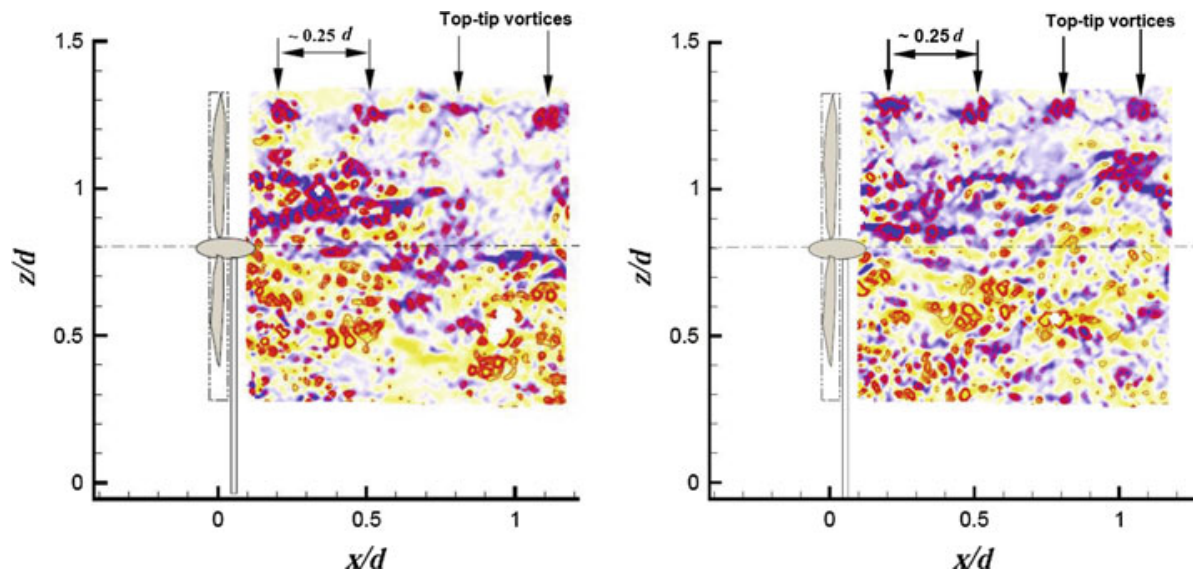

Fig. 7 Tip vortex structures shown with the spanwise vorticity $\left(\omega_{y}\right)$ overlaid with the swirling strength $\left(\lambda_{c i}\right)$ in instantaneous velocity fields at $x / d=0.2-1.2$. Neutral boundary layer (left) and the CBL (right)

\subsection{Behaviour of Tip Vortices}

Wind turbines induce a complex wake vortex system, including coherent helicoidal tip vortices (Vermeer et al. 2003, Derob et al. 2008). These vortices are associated with an enhanced turbulence level, noise generation and structural fatigue due to vortex induced vibration. We identified the coherent vortex structures using both the spanwise vorticity $\left(\omega_{y}\right)$ and the swirling strength $\left(\lambda_{c i}\right)$ distribution (Zhou et al. 1999; Adrian et al. 2000), which was described in Zhang et al. (2012). Here we concentrate on understanding how the CBL affects the behaviour of tip vortices.

Top-tip vortices can be seen immediately downwind of the turbine $(x / d=0.2-1.2)$ in instantaneous velocity fields (Fig. 7). The peaks of spanwise vorticity overlaid with the swirling strength indicate the vortex core locations. In the two examples, four tip vortices are visible at the top-tip height $(z / d=1.3)$, and there is no detectable coherent vortex structure 
displayed at the bottom-tip height $(z / d=0.3)$. The top-tip vortices are very similar to those reported in Dobrev et al. (2008) and visualized by smoke-wire visualization in Vermeer (2001). In both stability cases, the top-tip vortices persist up to about two rotor diameters downwind of the turbine.

Spectral analysis was performed using velocity fluctuations ( $u^{\prime}$ and $\left.w^{\prime}\right)$ to examine the distribution of energy across a range of frequencies in the wake. The analysis reveals the classical production subrange and inertial subrange of surface-layer turbulence, which follow power-law scaling with -1 and $-5 / 3$ slopes, respectively. More importantly, there are distinct localized high energy signatures at a frequency corresponding to coherent structures, including the tip vortices and large-scale wake meandering motions. Power spectra of $u^{\prime}$ and $w^{\prime}$ at the top-tip height are shown at $x / d=0.5,1.5$ and 3 for both the neutral and the CBL cases (Fig. 8). At $x / d=0.5$, multiple spectral peaks are found, i.e., the primary frequency of the top-tip vortex shedding $\left(3 f_{t}\right)$, the first $\left(6 f_{t}\right)$ and second $\left(9 f_{t}\right)$ harmonic frequencies $\left(f_{t}\right.$ is the rotation frequency of the rotor). With increasing downwind distance, the signature of the harmonic frequency is reduced and the magnitude becomes less, due to dissipation and the breaking-down of the tip vortices. At $x / d=1.5$, only the primary spectral peak is present for both cases. Further downwind at $x / d=3$, no peak is found. The enhanced energy at scales associated with tip vortex shedding persist farther downwind in the convective case, particularly for the streamwise component, which is also seen in enhanced turbulence intensity (addressed in the following section). In both the neutral and CBL cases, no frequency peak is detected at the bottom-tip level, where the turbulence level of the incoming flow is higher than that at the top-tip height and the wake may be affected by the presence of the ground (Fig. 9). This is also supported by S-PIV results. In addition, the overall wake meandering indicated by a distinct low frequency signature of $6 \mathrm{~Hz}$ can be seen in both cases, which is not affected by convective conditions. However, there is indeed more energy in the large production scales corresponding to the enhanced vertical transport in the CBL than in the neutral case, as illustrated in Figs. 8 and 9.

\subsection{Turbulence Intensity in the Wake}

Besides the velocity deficit, the other critical aerodynamic factor in wind-turbine wakes is the enhancement of turbulence intensity that is highly related to failure of wind-turbine components. Figure 10 compares the streamwise turbulence intensity $\left(I_{u}\right)$ in the centre plane $(y=0)$ of the wake for the neutral and CBL cases. High turbulence level occurs at the shear layers, immediately behind the nacelle for both cases, caused by hub/root vortices as reported in other wind-tunnel studies (Dobrev et al. 2008; Sherry et al. 2010). However, there is a remarkable difference in the turbulence intensity at the lower shear layer $(z / d=0.6)$, which displays greatly enhanced turbulence in the CBL than that in the neutral boundary layer. This is primarily related to the convective condition, under which the higher turbulence intensity of the inflow above the surface promotes turbulent mixing in the lower part of the wake.

Quantitative comparison of the turbulence intensity distribution calculated from hot-wire anemometry data in the central plane of the wake is shown in Fig. 11. The vertical profile of $I_{u}$ exhibits a "dual-peak" pattern at $x / d=2,3$ and 5 in both convective and neutral cases: a stronger primary peak at the top-tip level and the secondary peak below the hub height. It is not surprising to see that the first peak of $I_{u}$ is similar at $x / d=2$ for both neutral and convective cases, since the near-wake region is dominated by mechanical shear. The secondary peak of $I_{u}$ in the CBL is about $15 \%$ higher compared to that in the neutral boundary layer, which is consistent with S-PIV data. Furthermore, a large difference in $I_{u}$ is found at $x / d=3$. At this location, $I_{u}$ is approximately $20 \%$ higher in the CBL than that in the neutral boundary layer. 

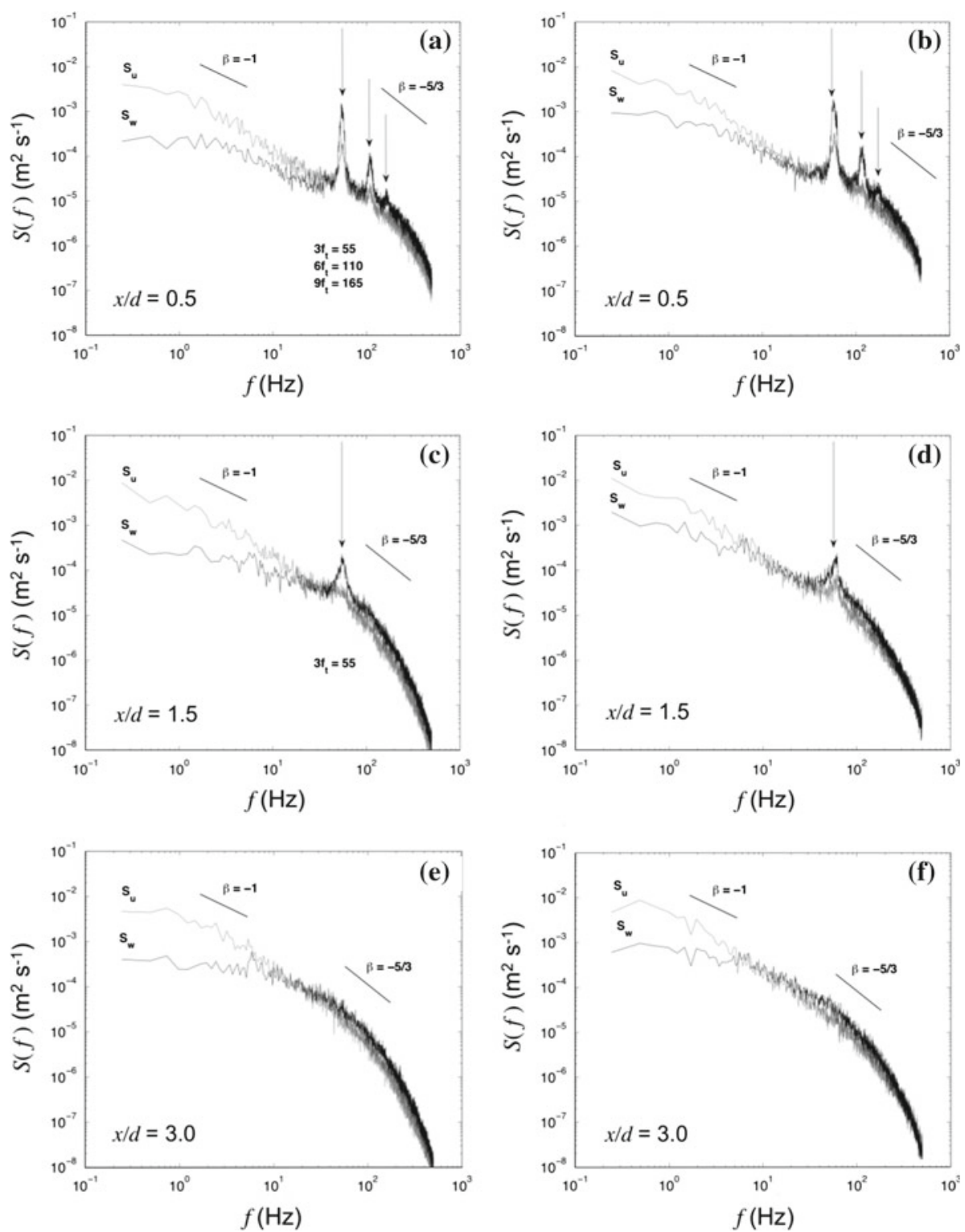

Fig. 8 Power spectra of $u^{\prime}$ and $w^{\prime}$ at the top-tip height in the centre plane of the wake $(y=0)$ at selected downwind locations. a, $\mathbf{b} x / d=0.5, \mathbf{c}, \mathbf{d} x / d=1.5$ and $\mathbf{e}, \mathbf{f} x / d=3.0$. Neutral boundary layer (left column) and CBL (right column)

This pronounced more intense turbulence is attributed to the higher shear production of TKE near the top-tip level and stronger turbulent transport in the lower part of the wake from the positive buoyancy effects. Further downwind in the wake, $I_{u}$ is reduced rapidly owing to the turbulent mixing. Therefore, the difference of $I_{u}$ between two stability cases is reduced in the upper part of the wake at $x / d=5$, while still apparent near the surface. Beyond $x / d=5$ the two peaks merge into a broad high value region in the middle of the wake. As a result, a 

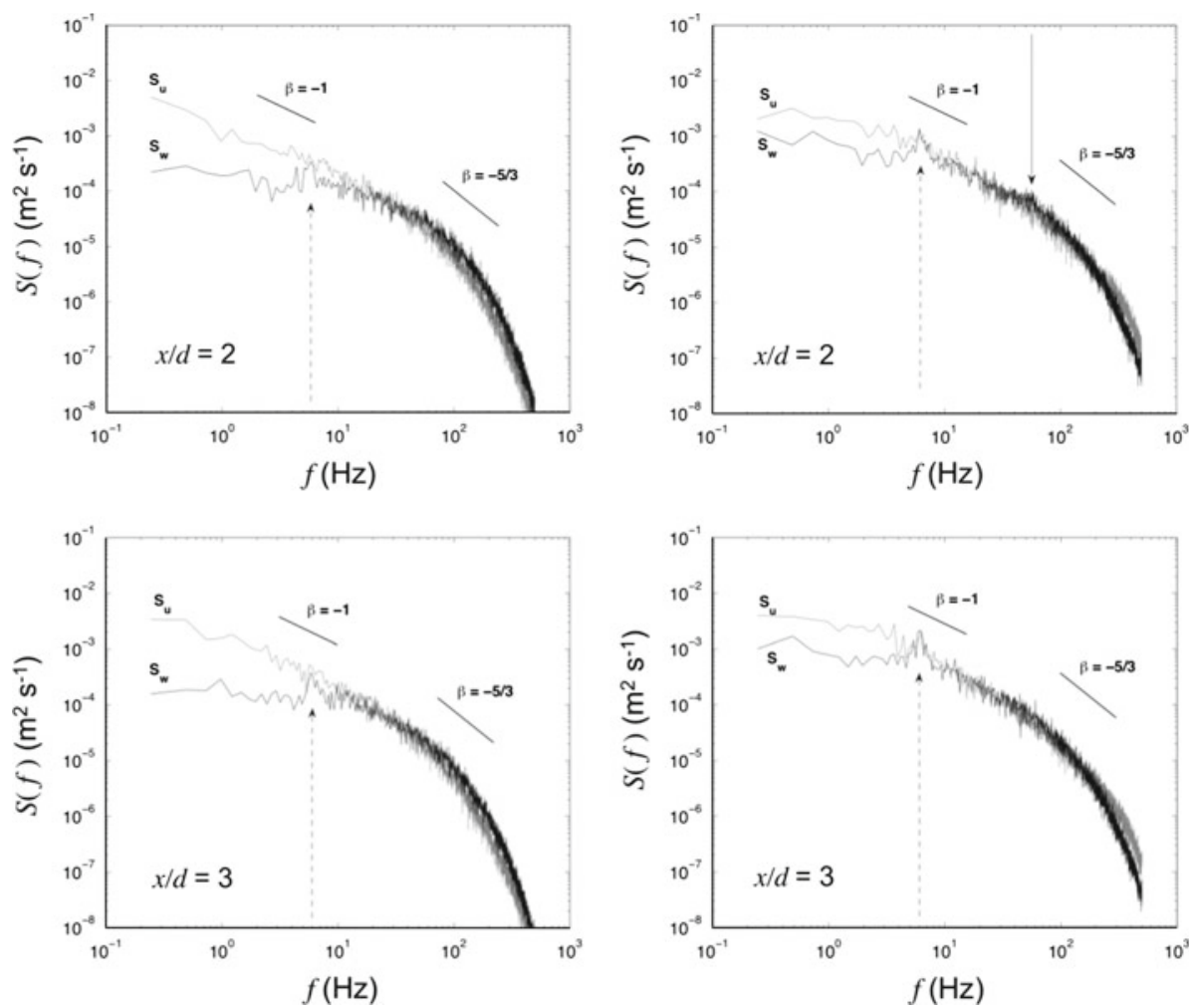

Fig. 9 Power spectra of $u^{\prime}$ and $w^{\prime}$ at the bottom-tip height at $x / d=2$ and 3 in the centre plane of the wake $(y=0)$. neutral boundary layer (left column) and CBL (right column)

more uniform vertical distribution of $I_{u}$ is observed at $x / d=10$. It should be noted that, in the far wake, the difference of $I_{u}$ between the two stability cases only remains in the vicinity of the surface, where the thermal effects are strongest. A similar turbulence structure with dual-peak turbulence intensity has been reported in other field and wind-tunnel studies. For example, Högström et al. (1988) observed that the turbulence intensity reached the maximum at about the top-tip and bottom-tip levels and two rotor diameters downwind of the wind turbine, and this dual-peak turbulence distribution remained until approximately four rotor diameters. The overall streamwise turbulence is caused by several coupled mechanisms, including the background/ambient boundary-layer turbulence $\left(I_{u 0}\right)$, turbulence generated by the shear layer of the wake, and turbulence created by the positive buoyancy.

It would be useful to understand how the added turbulence intensity $\left(I_{u+}=\sqrt{I_{u}^{2}-I_{u 0}^{2}}\right)$ in the wake varies in a CBL compared to that in a neutral boundary layer. Here we calculate the enhancement (or suppression) of turbulence intensity with respect to its counterpart of the inflow. For the flow regime of $I_{u} \geq I_{u 0}, I_{u+}=\sqrt{I_{u}^{2}-I_{u 0}^{2}}$; otherwise, $I_{u+}=\sqrt{I_{u 0}^{2}-I_{u}^{2}}$. As shown in Fig. 11, the distribution of added turbulence intensity $I_{u+}$ in the wake demonstrates pronounced differences in two respects. Firstly, at $x / d=2,3$ and $5, I_{u+}$ shows a distinct peak at roughly the top-tip height in both cases. In particular, the maximum added turbulence intensity occurs at $x / d=3$ and is approximately 0.17 in the CBL versus 0.12 in the neutral 

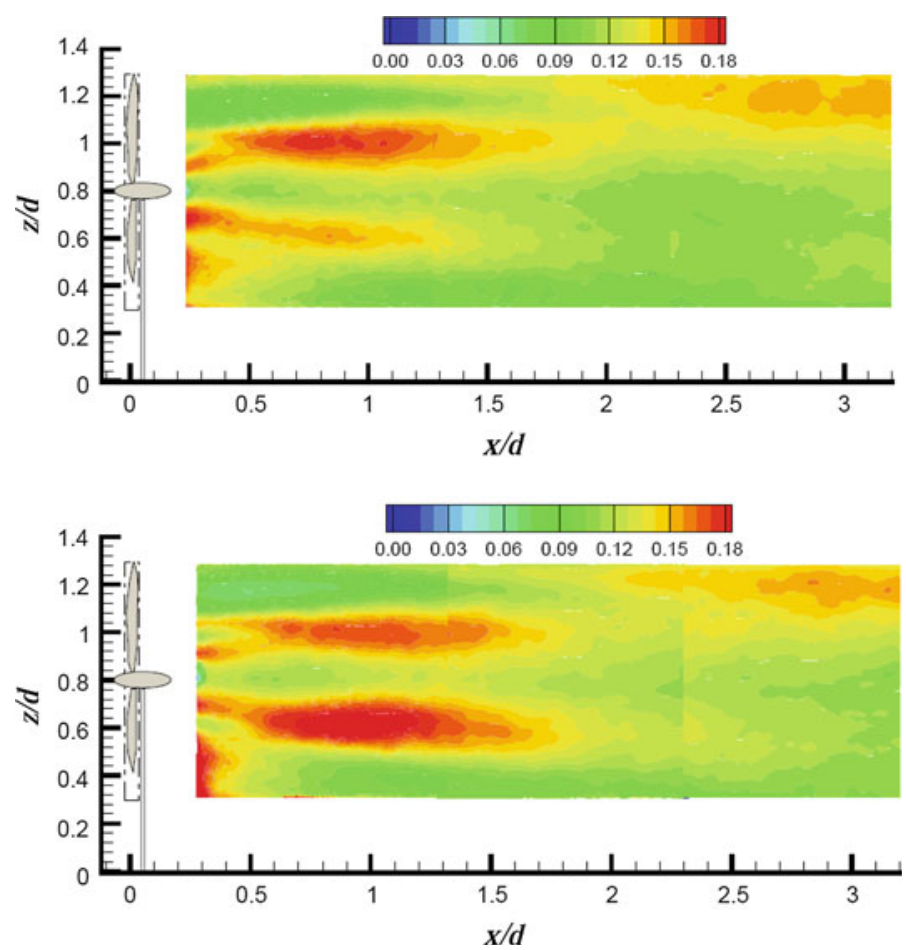

Fig. 10 The streamwise turbulence intensity $I_{u}\left(\sigma_{u} / U_{\text {hub }}\right)$ in the centre plane of the wake $(y=0)$ in the neutral boundary layer (upper) and the CBL (lower)

boundary layer. These maxima are related to the high shear, associated TKE production and possibly breaking down of the top-tip vortices. Secondly, similar to the $I_{u}$ distribution, in the lower part of the wake, the added turbulence is consistently higher in the CBL than in the neutral case. With increasing downwind distance, the difference of $I_{u+}$ between two stability cases remains near the surface, indicating that it is mainly caused by the effects of positive buoyancy, since the mechanical mixing from the wind turbine is reduced. In addition, we note that, below the bottom-tip height, turbulence is enhanced in the CBL case, whereas it is suppressed in the neutral case. This enhancement of turbulence is again attributed to the increased vertical transport due to surface heating.

\subsection{Turbulent Fluxes}

In addition to converting kinetic energy of the incoming flow to electricity and generating turbulence, a wind turbine mixes the air in the wake with the ambient air. The mixing affects the vertical transport of momentum, moisture and heat in the wake. The normalized momentum flux $\overline{u^{\prime} w^{\prime}} / U_{\text {hub }}^{2}$ is compared for the neutral and CBL cases (Fig. 12). These profiles are similar in two aspects at $x / d=2$ and 3 for both cases: the momentum flux has the opposite sign with respect to the rotor axis (negative above and positive below), which is due to the momentum transport between the large momentum "sink" in the wake centre and the ambient air. Moreover, the momentum flux reaches its maximum at the top-tip height, at $x / d=3$, due to strong shear, and the magnitude is approximately double that of the lower 

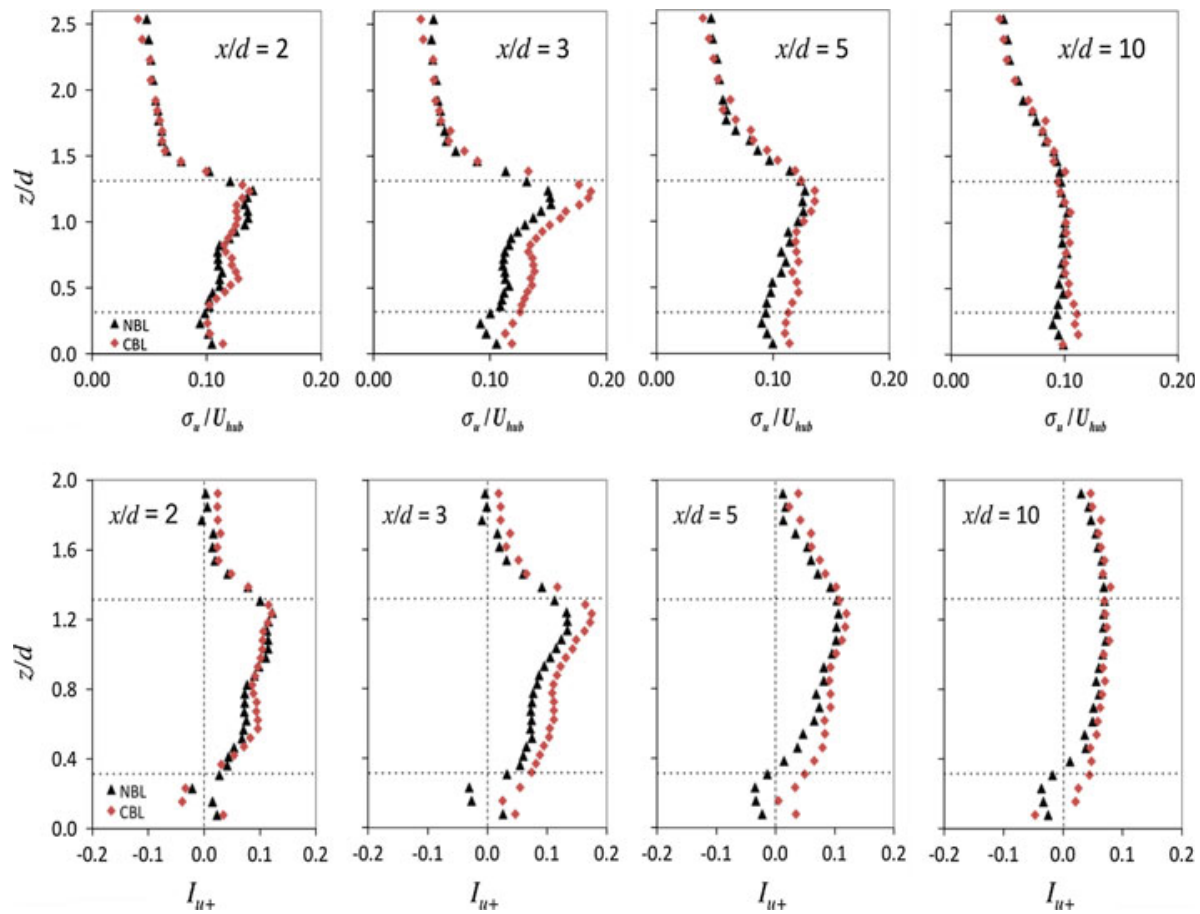

Fig. 11 Streamwise turbulence intensity $I_{u}$ (upper) and the added streamwise turbulence intensity $I_{u+}($ lower $)$ at $x / d=2,3,5$ and $10(y=0)$ in the neutral boundary layer and the convective boundary layer
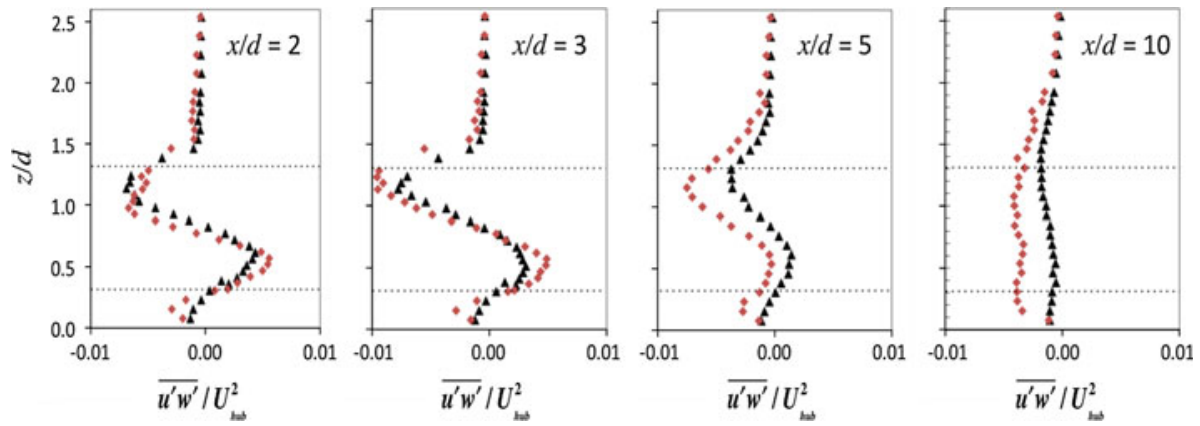

Fig. 12 Normalized momentum flux $\overline{u^{\prime} w^{\prime}} / U_{\text {hub }}^{2}$ at $x / d=2,3,5$ and $10(y=0)$ in the neutral and the convective cases

peak. However, the effects of positive buoyancy are evidently shown by the larger magnitude of the momentum flux in the lower part of the wake in the CBL compared to the neutral case. The results illustrate that, as a wind turbine operates in a CBL, the maximum momentum flux is at the top-tip level, while the second peak of the momentum flux occurs in the lower part of the wake, owing to an enhanced vertical transport caused by the positive buoyancy. This strong mixing and entrainment of cooler air from higher to lower altitude yields a cooling effect near the surface. Accordingly, the temperature of the lower part of the wake is lower than that of the inflow, as can be seen in Fig. 13a. Figure 13b shows that the kinematic heat flux $\overline{w^{\prime} \theta^{\prime}} /\left[U_{\text {hub }}\left(\theta_{s}-\theta_{0}\right)\right]$ is primarily affected at $x / d=2$ and 3 in the centre plane of the 

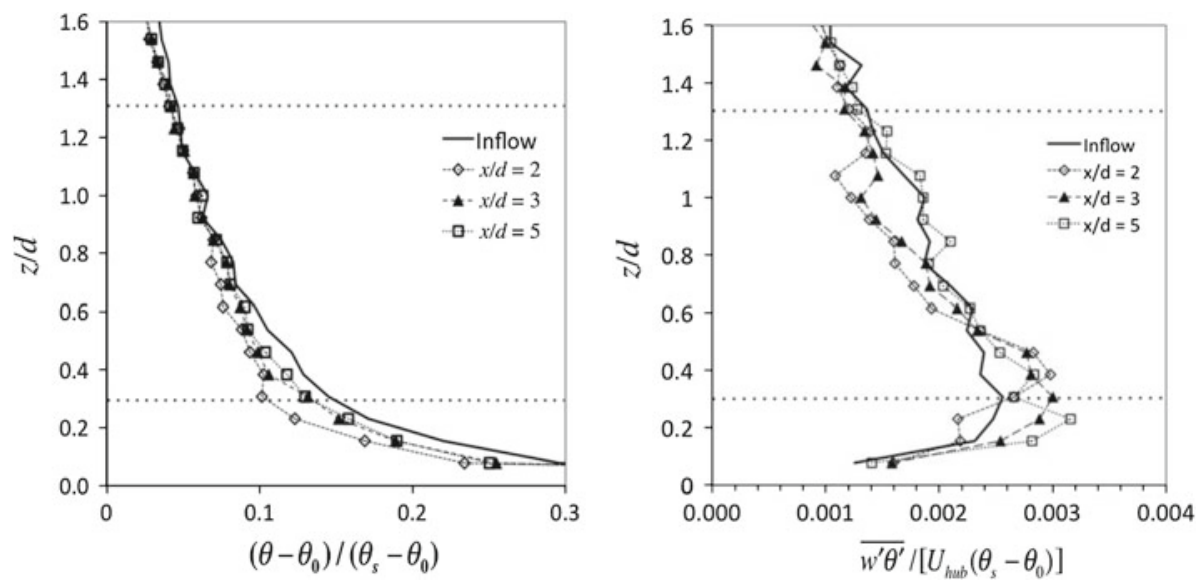

Fig. 13 The mean temperature $\left(\theta-\theta_{0}\right) /\left(\theta_{s}-\theta_{0}\right)$ and turbulent kinematic heat flux $\overline{w^{\prime} \theta^{\prime}} /\left[U_{\text {hub }}\left(\theta_{s}-\theta_{0}\right)\right]$ profiles at $x / d=2,3$ and $5(y=0)$ in the CBL case, compared with the incoming flow

wake. In this region, the turbulent kinematic heat flux displays a distinct deviation from that of the incoming flow. Heat flux is particularly enhanced by about $25 \%$ at the lower part of the wake, consistent with the trend of the turbulent momentum flux. Also, the reduction of temperature below the hub height (Fig. 13a) results in a larger temperature gradient near the surface, which is consistent with the enhanced heat flux.

\section{Summary}

Wind-tunnel experiments were performed to study the convective boundary-layer effects on wind-turbine wakes. Although lacking a capping inversion layer, the simulated CBL reasonably represents the lower portion (up to $z / \delta=0.4$ ) of a classic overland CBL. Since the wind-turbine model was limited to the lowest one third of the boundary-layer depth, this study enabled us to investigate surface-layer buoyancy effects on the structure of wind-turbine wakes.

Both S-PIV and customized triple-wire anemometry were used to characterize the mean wind velocity, tip vortices, velocity spectra, turbulence intensity and turbulent fluxes of the wake up to a downwind distance of $x / d=20$ in the CBL versus the neutral case. The velocity deficit is smaller in the convective compared to the neutral case, particularly in the lower part of the wake, due to enhanced radial momentum transport under the convective conditions. The velocity deficit at the wake centre is about $15 \%$ less in the CBL case, and its decay follows a power law (with a slope of -0.8) in both cases.

Helicoidal tip vortices, shed at the frequency of consecutive blades $\left(3 f_{t}\right)$, are observed at the top-tip level and persist up to a downwind distance of about two rotor diameters, regardless of thermal stability. Tip vortices are not detected at the bottom-tip level owing to the higher levels of background turbulence, which dissipates them faster. More energy is found in the large production scales of the velocity spectra in the convective case than that in the neutral case, due to enhanced vertical motions.

The maximum of turbulence intensity occurs roughly at the top-tip level and $x / d=3$, and the magnitude is $20 \%$ higher in the CBL than that in the neutral boundary layer. This 
maximum of the turbulence intensity is related to the high shear, associated TKE production and likely the breaking-down of the top-tip vortices. Also, it is noticeable that turbulence is enhanced in the lower part of the wake in the CBL case, due to the promotion of vertical transport of momentum by higher incoming turbulence intensity caused by positive buoyancy. With increasing downwind distance, the effects of positive buoyancy on the flow remain evident near the surface while the effects of mechanical mixing from the rotor are reduced gradually.

The magnitude of negative momentum flux is largest at the top-tip level and $x / d=3$, whereas the second largest magnitude is in the lower part of the wake due to the effect of positive buoyancy in the CBL case. Strong mixing and entrainment of cooler air from above results in a cooling effect near the surface. In addition, turbulent kinematic heat flux is strongly enhanced by about $25 \%$ in the lower part of the wake, compared to the undisturbed CBL inflow.

Overall, the CBL results in a smaller velocity deficit and enhanced turbulence both at the top-tip level and the lower part of the wake, compared to the neutral case. The dataset presented here was obtained in the centre plane of a stand-alone wind-turbine wake under a relatively weak CBL condition $\left(R i_{b}=-0.13\right)$. More remarkable effects may be expected in the field with stronger convection. This unique dataset will be used for the validation of high-resolution numerical models (e.g., LES) of wind-turbine wakes in a CBL, similar to that performed by Wu and Porté-Agel (2011) in a neutral boundary layer. Future work will also address the effects of atmospheric stability on the wind-turbine wakes in wind farms, i.e., the interaction of multiple wakes with the ABL. Additionally, it would be of interest to look into the combined effects of atmospheric stability and topography on the wind-turbine wakes since both effects are often coupled in the field.

Acknowledgments This research was supported by the Swiss National Foundation (Grant 200021-132122), the National Science Foundation (Grant ATM-0854766) and NASA (Grant NNG06GE256). C. Markfort would like to acknowledge funding from NSF IGERT (Grant DGE-0504195) and NASA Earth and Space Science Fellowship (Grant NNX10AN52H). Thanks also go to the research engineer Jim Tucker for his efforts in preparation of the experimental facility and instruments. Computing resources were provided by the University of Minnesota Supercomputing Institute and the Swiss National Supercomputing Centre under project ID s306.

\section{References}

Adrian RJ, Christensen KT, Liu ZC (2000) Analysis and interpretation of instantaneous turbulent velocity fields. Exp Fluids 29:275-290

Baker RW, Walker SN (1984) Wake measurements behind a large horizontal axis wind turbine generator. Solar Energy 33:5-12

Barthelmie R, Larsen G, Pryor S, Jorgensen H, Bergstrom H, Schlez W, Rados K, Lange B, Volund P, Neckelmann S, Mogensen S, Schepers G, Hegberg T, Folkerts L, Magnusson M (2004) ENDOW (efficient development of offshore wind farms): modelling wake and boundary layer interactions. Wind Energy 7:225-245

Cal RB, Lebrón J, Castillo L, Kang HS, Meneveau C (2010) Experimental study of the horizontally averaged flow structure in a model wind-turbine array boundary layer. J Renew Sustain Energy 2:013,106

Carper MA, Porté-Agel F (2008) Subfilter-scale fluxes over a surface roughness transition Part I: measured fluxes and energy transfer rates. Boundary-Layer Meteorol 126:157-179

Chamorro LP, Porté-Agel F (2009) A wind-tunnel investigation of wind-turbine wakes: boundary-layer turbulence effects. Boundary-Layer Meteorol 132(1):129-149

Chamorro LP, Porté-Agel F (2010) Thermal stability and boundary-layer effects on wind-turbine wakes: a wind-tunnel study. Boundary-Layer Meteorol 136:515-533

Deardorff JW (1970) Convective velocity and temperature scales for the unstable planetary boundary layer and for Raleigh convection. J Atmos Sci 27:1211-1213

Dobrev I, Maalouf B, Troldborg N, Massouh F (2008) Investigation of the wind turbine vortex structure. In: 14th international symposium on applications of laser techniques to fluid mechanics, Lisbon, Portugal 
Fedorovich E, Kaiser R, Rau M, Plate E (1996) Wind tunnel study of turbulent flow structure in the convective boundary layer capped by a temperature inversion. J Atmos Sci 53:1273-1289

Fedorovich E, Nieuwstart FTM, Kaiser R (2001a) Numerical and laboratory study of a horizontally evolving convective boundary layer. Part I: Transition regimes and cevelopment of the mixed Layer. J Atmos Sci 58:70-86

Fedorovich E, Nieuwstart FTM, Kaiser R (2001b) Numerical and laboratory study of a horizontally evolving convective boundary layer. Part II: effects of elevated wind shear and surface roughness. J Atmos Sci 58:546-560

Frandsen S, Barthelmie R, Pryor S, Rathmann O, Larsen S, Hojstrup J, Thogersen M (2006) Analytical modelling of wind speed deficit in large offshore wind farms. Wind Energy 9:39-53

Garratt JR (1994) The atmospheric boundary layer. Cambridge University Press, U.K., 316 pp

Hancock PE, Pascheke F (2010) Wind tunnel simulations of wind turbine wake interactions in neutral and stratified wind flow. In: 10th EMS annual meeting, 10th European conference on applications of meteorology (ECAM), Sept 13-17, 2010 in Zurich, Switzerland

Högström U, Asimakopoulos DN, Kambezidis H, Helmis CG, Smedman A (1988) A field study of the wake behind a 2 MW wind turbine. Atmos Environ 22(4):803-820

Hutchins N, Nickels TB, Marusic I, Chong MS (2009) Hot-wire spatial resolution issues in wall-bounded turbulence. J Fluid Mech 635:103-136

Jorgensen FE (1996) The computer-controlled constant temperature ameometer: aspectes of the set-up, probe calibration, data acquisition, and data collection turbulence. Meas Sci Technol 12:1378-1387

Lebrón J, Castillo L, Cal RB, Kang HS, Meneveau C (2010) Interaction between a wind turbine array and a turbulent boundary layer. In: 48th AIAA aerospace sciences meeting including the new horizons forum and aerospace exposition 4-7 January 2010, Orlando, Florida

Magnusson M, Smedman AS (1994) Influence of atmospheric stablity on wind turbine wakes. Wind Energy 18:139-152

Magnusson M, Smedman AS (1999) Air flow behind wind turbines. J Wind Eng Ind Aerodyn 80:169-189

Medici D, Alfredsson PH (2006) Measurements on a wind turbine wake: 3D effects and bluff body vortex shedding. Wind Energy 9:219-236

Meroney RN, Melbourne WH (1992) Operating ranges of meteorological wind tunnels for the simulation of convective boundary layer (CBL) phenomena. Boundary-Layer Meteorol 61:145-174

Ohya Y (2001) Wind-tunnel study of atmospheric stable boundary layers over a rough surface. BoundaryLayer Meteorol 98:57-82

Ohya Y, Uchida T (2004) Laboratory and numerical studies of the convective boundary layer capped by a strong inversion. Boundary-Layer Meteorol 112:223-240

Porté-Agel F, Lu H, Wu YT, Conzemius RJ (2011) Large-eddy simulation of atmospheric boundary layer flow through wind turbines and wind farms. J Wind Eng Ind Aerodyn 99:154-168

Sherry M, Sheridan J, Jacono DL (2010) Horizontal axis wind turbine tip and root vortex measurements. In: 15 th international symposium on applications of laser techniques to fluid mechanics, Lisbon, Portugal, 5-8 July

Sørensen JN (2011) Aerodynamic aspects of wind energy conversion. Annu Rev Fluid Mech 43:427-448

Stull R (1988) An introduction to boundary-layer meteorology. Kluwer Academic Publishers, Dordrecht, 666 $\mathrm{pp}$

van den Berg GP (2008) Wind turbine power and sound in relation to atmospheric stability. Wind Energy 11:151-169

Vermeer LJ (2001) A review of wind turbine wake research at TUDelft. In: Proceedings of ASME Wind Energy Symposium, ASME, New York, AIAA-2001-0030, vol 39, pp 103-113

Vermeer LJ, Sørensen JN, Crespo A (2003) Wind turbine wake aerodynamics. Prog Aero Sci 39:467-510

Whale J, Papadopoulos KH, Anderson CG, Helmis CG, Skyner DJ (1997) A study of the near wake structure of a wind turbine comparing measurements from laboratory and full-scale experiments. Solar Energy 56:621-633

Whale J, Anderson CG, Bareiss R, Wagner S (2000) An experimental and numerical study of the vortex structure in the wake of a wind turbine. J Wind Eng Ind Aerodyn 84:1-21

Wharton S, Lundquist JK (2010) Atmospheric stability impacts on power curves of tall wind turbines an analysis of a west coast North American wind farm. LLNL-TR-424425

Wu YT, Porté-Agel F (2011) Large-eddy simulation of wind-turbine wakes: evaluation of turbine parametrisations. Boundary-Layer Meteorol 132:129-149

Zhang W, Markfort CM, Porté-Agel F (2012) Near-wake flow structure downwind of a wind turbine in a turbulent boundary layer. Exp Fluids 52:1219-1235. doi:10.1007/s00348-011-1250-8

Zhou J, Adrian RJ, Balachandar S, Kendall TM (1999) Mechanisms for generating coherent packets of hairpin vortices in channel flow. J Fluid Mech 387:353-359 